\title{
重载铁路大跨度钢桁梁桥复合材料轨枕适应性
}

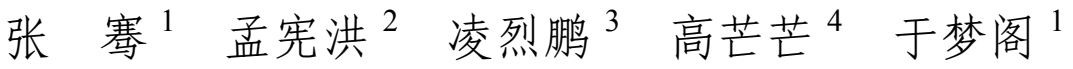 \\ (1. 青岛大学机电工程学院 青岛 266071; \\ 2. 朔黄铁路发展有限责任公司 北京 100038 ; \\ 3. 中国铁道科学研究院铁道建筑研究所 北京 100081 ; \\ 4. 中国铁道科学研究院基础设施检测研究所 北京 100081)
}

\begin{abstract}
摘要: 我国铁路钢桥传统上采用木枕传递活载, 尽管木材有着良好的弹性和绝缘性能, 但存在木材用量大和养护维修繁琐的 不足, 复合材料轨枕可通过截面设计使其在外形和重量上与木枕相近。为研究复合材料轨枕在 $30 \mathrm{t}$ 轴质量重载铁路上的适用 性, 对采用木枕和复合材料轨枕的 $64 \mathrm{~m}$ 单线铁路栓焊下承析梁进行动力响应分析, 考察货车以不同速度通过桥梁时车辆、 轨枕和桥梁的动力性能, 对比分析木枕和复合材料轨枕的受力和变形。研究结果表明: 铺装不同类型轨枕时, 就桥梁而言, 各车速下跨中垂向位移和加速度均低于规范限值, 满足行车安全要求; 就车辆而言, 各车速下轮重减载率和车体振动也均满 足要求, 且铺装木枕或复合材料轨枕时系统动力响应无显著差异; 复合材料轨枕的应力比木枕大, 但变形小于木枕, 无论应 力还是变形均远小于复合材料轨枕的许用应力和许用变形; 就复合材料轨枕而言, 虽然距力作用点较近处的轨枕受载比木枕 大, 但是两者差距小于 $2 \%$, 可认为基本一致。复合材料轨枕能满足 $30 \mathrm{t}$ 轴质量货车在 $60 \sim 90 \mathrm{~km} / \mathrm{h}$ 范围内安全平稳运行的 要求, 适合作为木枕替代品。
\end{abstract}

关键词: 钢析梁; 复合材料轨枕; 车-线-桥耦合振动; 行车安全; 平稳性

中图分类号: U214

\section{Adaptability of Composite Material Sleeper for Heavy Railway Steel Truss Girder Bridge}

\section{ZHANG Qian $^{1} \quad$ MENG Xianhong $^{2} \quad$ LING Liepeng $^{3} \quad$ GAO Mangmang $^{4} \quad$ YU Mengge $^{1}$}

(1. Mechanical and Electrical Engineering College, Qingdao University, Qingdao 266071;

2. Shuohuang Railway Development Co., Ltd, Beijing 100038;

3. Railway Engineering ResearchInstitute, China Academy of Railway Sciences, Beijing 100081;

4. Infrastructure Inspection Research Institute, China Academy of Railway Sciences, Beijing 100081)

\begin{abstract}
Wooden sleepers are traditionally used to transmit live loads on railway steel bridges in China. Although wood has good elasticity and insulation properties, it has the disadvantages of large amount of wood and cumbersome maintenance and repair. Composite sleepers can be similar in shape and weight to wooden pillows. Composite sleeperswhichcan be designed in terms of shape and weight are similar as wooden pillows. In order to study the applicability of the composite material sleeper on the $30 \mathrm{t}$ axle mass load railway,coupling vibration analysis of the $64 \mathrm{~m}$ single-wire railway underpinning with wooden pillow and composite material sleeper was carried out. At different speeds through the bridge to examine the dynamic performance of train and bridge. The results show that: in the case of different types of sleeper, the vertical displacement and acceleration of the train are lower than the standard limit for the bridge, and the traffic safety requirements are satisfied. For the train, wheel load reduction and body vibration are also required to meet the requirements, and pavement or composite pillow when the system dynamic response is no significant difference. The stress of the composite sleeper is larger than that of the pillow, but still far less than the allowable stress of the composite sleeper. The deformation of the composite sleeper is less than that of the pillow, the deformation is less than permissible deformation;for the
\end{abstract}

* 朔黄铁路发展有限责任公司科技基金(SHGF-14-51)、国家自然科学基金 (51705276)和青岛市博士后基金应用(40518060011)资助项目。20180614 收到初稿, 20181012 收到修改稿 
composite sleeper, although the load near the force point of the sleeper is bigger than the pillow, but the gap between the two is less than $2 \%$, basically the same.therefore, composite sleepers can meet the trainin the range of $60 \sim 90 \mathrm{~km} / \mathrm{h}$ safe and stable operation requirements and suitable as a substitute for wooden pillows.

Key words: steel truss girder; omposite material sleeper; train-track-bridge coupling vibration; safety; stability

\section{0 前言}

我国铁路钢桥传统上采用木枕传递活载, 并由 护木、护轨及有关的联结件组成明桥面。尽管木材 有着良好的弹性和绝缘性能, 但长期运营存在明显 缺陷, 首先是木材用量大, 每延米桥面约需木材 $0.4 \sim 0.5 \mathrm{~m}^{3}$, 且由于木材寿命周期短而需频繁更换, 其次是联结部位繁多，联结方式和作业项目相对繁 琐，而机械磨损或开裂又导致维修周期短, 大大增 加了养护维修工作量, 因此新建桥梁目前已基本采 用混凝土枕 ${ }^{[1-5]}$ 。然而对既有钢桥而言, 混凝土枕与 木枕在重量上的明显差异使得混凝土枕不能作为木 枕的替代品, 而复合材料轨枕可通过截面设计使其 在外形和重量上与木枕相近, 因而成为一项可行的 选择。

国内外学者在复合轨枕方面开展了一系列研 究。日本积水化学工业株式会社 ${ }^{[6]}$ 研制了一种复 合轨枕, 重点研究其耐久性; 日本铁路综合技术 研究所 ${ }^{[7]}$ 对复合轨枕进行了测试, 验证了其良好 的疲劳性能; 奥地利格拉茨工业大学 ${ }^{[8]}$ 对明桥面 钢桥上的合成轨枕进行了全生命周期成本 (LCC) 分析; 德国慕尼黑工业大学 ${ }^{[9]}$ 按照欧洲标准对复 合轨枕进行了试验研究; 美国铁路运输技术中心 监测复合轨枕的性能, 包括轨枕挠度和轨距 ${ }^{[10]}$, 美国伊利诺大学按照铁路工程和维护协会推荐的 试验方法研究了高密度聚乙烯轨枕, 结果表明在 荷载作用下其呈现脆性断裂的破坏模式 ${ }^{[11]}$; 我国
成都铁路局在沪昆上行线新水花大桥采用合成轨 枕替代劣化的木枕, 跟踪观测其使用性能; 北京 化工大学王守琛 ${ }^{[12]}$ 利用废弃高聚物通过双阶挤出 机试制复合材料轨枕; 东北林业大学陈玉霄 ${ }^{[13]}$ 从满 足抗压强度等角度出发, 设计了新型复合材料轨 枕。国内外学者以往的研究更多关注从材料、结 构、工艺的角度设计复合材料轨枕, 以及研究复 合材料轨枕的破坏模式等, 缺少对复合材料轨枕 的动力响应研究, 尤其是铺装在重载铁路上的适 应性研究。

本文以既有线钢桁梁桥明桥面上铺装的玻璃纤 维增强树脂复合材料轨枕(简称复合材料轨枕)为研 究对象, 与原有木枕进行对比分析, 考察复合材料 轨枕在重载铁路钢桥上的适应性, 主要包括两部分 内容, 第一部分通过车-线-桥动力仿真分析, 考察 复合材料轨枕替换木枕后对列车、线路和桥梁动力 响应的影响, 第二部分对比分析复合材料轨枕和木 枕的应力和变形。

\section{1 车-线-桥动力仿真模型}

该 $64 \mathrm{~m}$ 单线钢析梁桥材料为 $16 \mathrm{Mnq}$, 活载采 用中一 22 级。原桥上线路采用明桥面木枕, 受防爬 角钢影响, 桥上木枕并未均匀布置, 而是每 $8 \mathrm{~m}$ 节 间布置 23 根木枕，桥两端头各布置 2 根，共计 188 根。钢桥两侧过渡段设置了 14 根木枕, 其后采用 III 型混凝土轨枕。过渡段左右对称。端部及 $8 \mathrm{~m}$ 节间 内的木枕布置如图 1 所示。

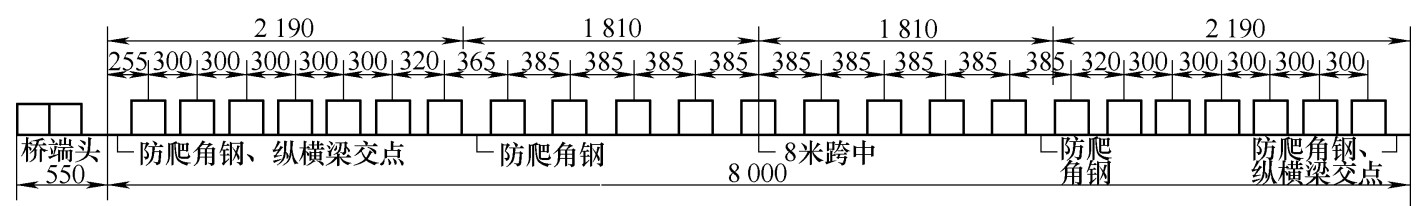

图 $18 \mathrm{~m}$ 节间内轨枕布置及桥端头 2 根枕布置

为了研究桥上木枕更换为复合材料轨枕后对车 线桥系统动力性能的影响, 对 $64 \mathrm{~m}$ 单线栓焊下承桁 梁桥及线路建立了车线桥动力分析模型, 其中线路 结构考虑了非均匀布置及轨枕变化的影响, 桥梁两 侧线路长度均须不小于列车长度和 20 倍轨枕间距 之和, 以消除钢轨端部边界效应的影响, 实际计算
中桥梁两侧线路均长 $187.23 \mathrm{~m}$ 。车辆则采用 $30 \mathrm{t}$ 轴 质量重载货车。

列车由多节机车和车辆组成。每节车辆都是由 车体、转向架、轮对、弹簧和阻尼组成的多自由度 垂向振动系统。从解决工程问题的角度出发, 对车 辆进行了以下假定 ${ }^{[14]}$ : (1) 车体、转向架和轮对均 
为刚体; (2) 列车在桥上作等速运动, 不考虑纵轴方 向动力作用的影响; (3) 车辆所有悬挂系统之间的阻 尼均为粘性阻尼, 所有弹簧均为线性弹簧; (4) 轮轨 之间法向力采用赫兹非线性弹性接触理论确定, 允 许轮轨相互脱离。

本文根据 $30 \mathrm{t}$ 轴质量货车的悬挂参数特点, 采 用两系悬挂车辆模型, 共 10 个自由度。车辆模型示 意图见图 2。计算工况列于表 1 。

表 1 车线桥耦合振动分析工况汇总

\begin{tabular}{ccccc}
\hline 名称 & 编组 & 轨枕 & $\begin{array}{c}\text { 计算车速/ } \\
(\mathrm{km} / \mathrm{h})\end{array}$ & $\begin{array}{c}\text { 轨道 } \\
\text { 不平顺 }\end{array}$ \\
\hline $\begin{array}{c}\text { 轴质量 } 30 \mathrm{t} \\
\text { 重载货车 }\end{array}$ & 10 辆编组 & $\begin{array}{c}\text { 木枕 } \\
\text { 复合材料 } \\
\text { 轨枕 }\end{array}$ & $\begin{array}{c}60,70, \\
80,90\end{array}$ & $\begin{array}{c}\text { 美国五级 } \\
\text { 谱样本 }\end{array}$ \\
\hline
\end{tabular}

轨道结构是车-线-桥动力学系统中的一个重要 组成部分, 一方面它承受着车辆施加的力, 同时又 将所受到的力传递给桥梁, 使桥梁产生振动和变形;
另一方面它又把桥梁的振动和变形传递给车辆, 影 响车辆的动力性能。动力分析时对轨道结构采用连 续分布参数多层离散点支承梁模型, 即钢轨被视为 连续弹性离散点支承上的无限长 Euler 梁, 轨下基 础沿纵向被离散。离散以各轨枕支点为基元, 每个 基元的钢轨与轨枕以及轨枕与桥面之间通过弹簧与 阻尼连接。图 3 为明桥面轨道动力学模型。

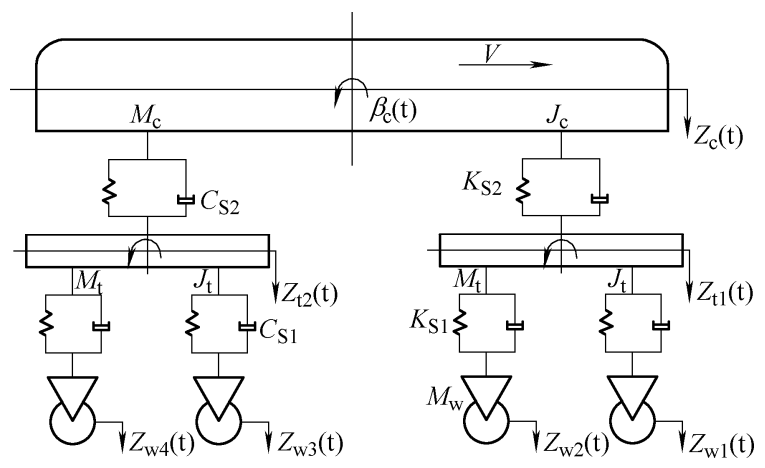

图 2 具有二系悬挂的车辆模型

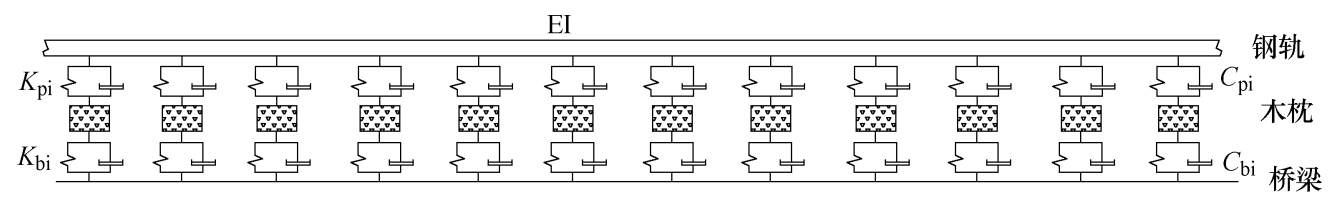

图 3 明桥面轨道动力学模型

钢桥明桥面木枕采用分开式 $\mathrm{K}$ 形扣件连接, 复 合材料轨枕采用弹条 II 型扣件连接, 线路弹性主要 由轨枕提供, 复合材料轨枕的截面尺寸如图 4 所示。 进行动力性能分析时钢轨和轨枕弹性连接, 轨枕模 拟为带质量刚体, 其弹性通过轨枕与桥面之间的弹 性连接来表述。现有资料未能直接提供木枕和复合 材料轨枕的弹性, 为此, 采用有限元软件 MIDAS 建模分析确定其等效刚度。复合材料轨枕和木枕均 采用实体单元建模, 枕木与钢梁在其接触面范围内 以简支形式连接, 在铁垫板对应的范围内施加面荷 载, 木枕和复合材料轨枕的等效刚度计算示意图见 图 5, 得到桥上木枕的等效刚度为 $170 \mathrm{MN} / \mathrm{m}$, 复合 材料轨枕的等效刚度为 $187 \mathrm{MN} / \mathrm{m}$ 。

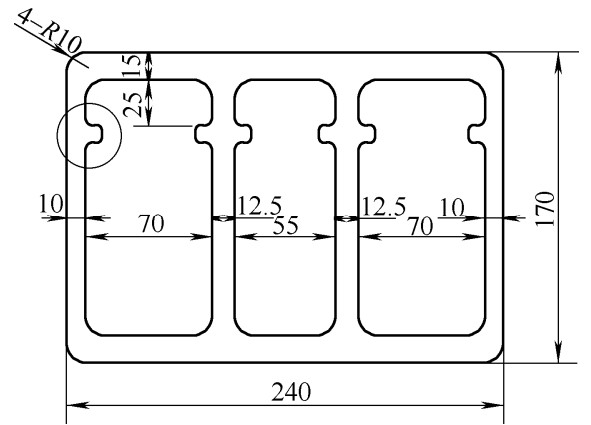

图 4 复合材料轨枕截面尺寸

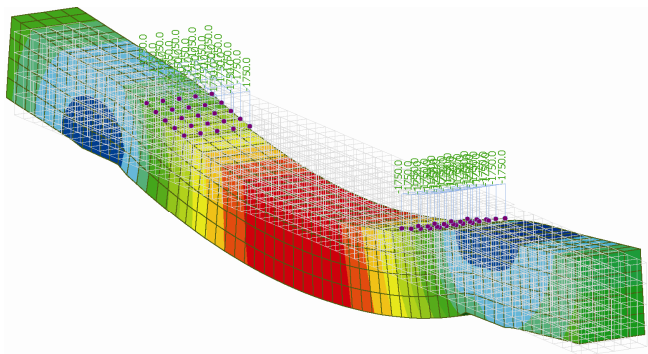

(a)木枕

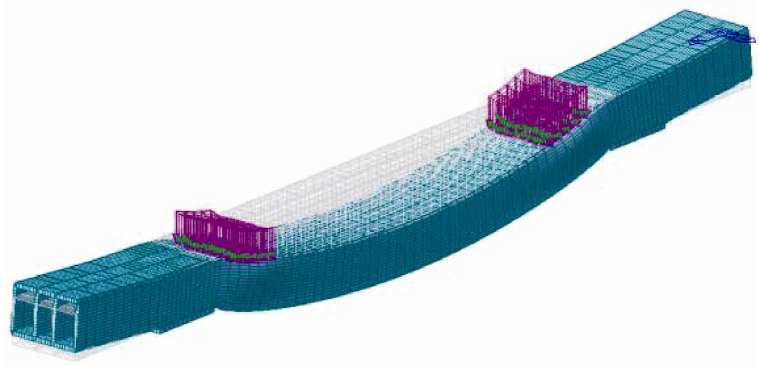

(b)复合材料轨枕

图 5 轨枕等效刚度计算示意图

桥枕材料及截面特性见表 2。

轨道不平顺是引起轮轨系统产生振动的主要根 源, 是轨道方面影响行车安全性和平稳舒适性的控 制因素。轨道不平顺对轮轨系统动力特性的影响是 通过对轮轨接触关系的改变而体现的。由于美国五 级谱与我国三大干线轨道谱大体相当, 允许货车速 
表 2 桥枕材料及截面特性表

\begin{tabular}{|c|c|c|c|}
\hline \multicolumn{2}{|c|}{ 桥枕类型 } & 木枕 & 复合材料轨枕 \\
\hline \multicolumn{2}{|c|}{ 外形尺寸 $(\mathrm{mm} \times \mathrm{mm} \times \mathrm{mm})$} & $3000 \times 220 \times 260$ & $3000 \times 240 \times 170$ \\
\hline \multicolumn{2}{|c|}{ 弯曲弹模 $E / \mathrm{GP}$} & 9.0 & 40.0 \\
\hline \multicolumn{2}{|c|}{ 等效刚度/(N/m) } & $1.7 \times 10^{8}$ & $1.87 \times 10^{8}$ \\
\hline \multirow{3}{*}{$\begin{array}{l}\text { 截面转动 } \\
\text { 惯量 } / \mathrm{m}^{4}\end{array}$} & 扭转 $J$ & $4.519 \times 10^{-4}$ & $5.966 \times 10^{-5}$ \\
\hline & 绕水平轴 $I_{1}$ & $3.222 \times 10^{-4}$ & $5.519 \times 10^{-5}$ \\
\hline & 绕垂直轴 $I_{2}$ & $2.307 \times 10^{-4}$ & $5.947 \times 10^{-5}$ \\
\hline
\end{tabular}

度达到 $128 \mathrm{~km} / \mathrm{h}$, 客车速度达到 $144 \mathrm{~km} / \mathrm{h}$, 因此计 算中采用美国五级谱生成的轨道不平顺时域样本, 轨道不平顺样本最大幅值列于表 3 。

表 3 轨道不平顺样本幅值

$\mathrm{mm}$

\begin{tabular}{ccccc}
\hline & $\begin{array}{c}\text { 左轨轨向 } \\
\text { 不平顺 }\end{array}$ & $\begin{array}{c}\text { 右轨轨向 } \\
\text { 不平顺 }\end{array}$ & $\begin{array}{c}\text { 左轨高低 } \\
\text { 不平顺 }\end{array}$ & $\begin{array}{c}\text { 右轨高低 } \\
\text { 不平顺 }\end{array}$ \\
\hline 美国五级谱 & 10.357 & 10.6873 & 13.7322 & 14.8668 \\
\hline
\end{tabular}

该轨道样本随距离的变化如图 6、7 所示。

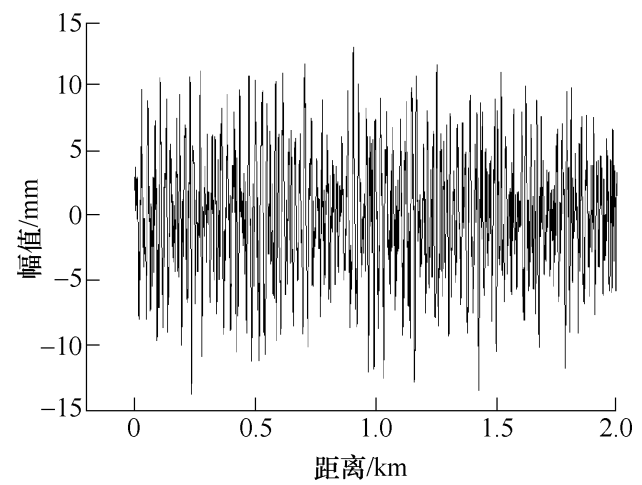

图 6 美国五级谱左轨高低轨道不平顺

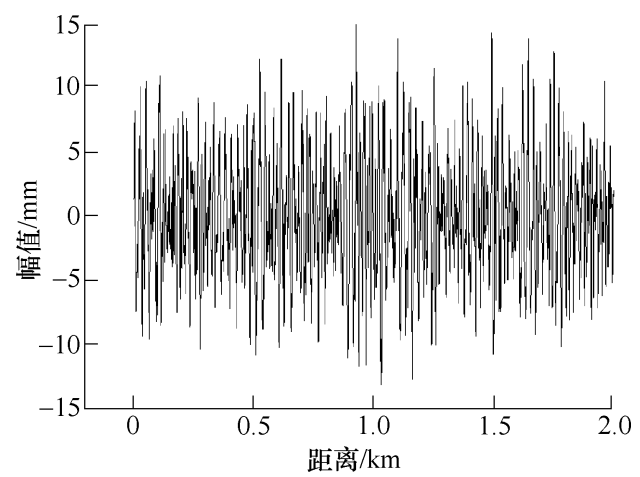

图 7 美国五级谱右轨高低轨道不平顺

桥梁各杆件均采用空间梁单元建模。对于桥梁 的二期荷载, 将其作为均布荷载质量分配到铁路纵 梁和下弦上。桥梁结构采用一致刚度矩阵和一致质 量矩阵, 阻尼矩阵采用瑞利阻尼模型, 即通过瑞利 阻尼系数表示成质量阵与刚度阵的线性组合, 其数 学表达式为

$$
C=\alpha M+\beta K
$$

钢桥阻尼比按 $1 \%$ 选取。桥梁有限元模型见图 8 。为了更准确地模拟桥面结构对车桥动力的影响, 考虑了两片纵梁之间的连接杆件, 如图 9 所示。

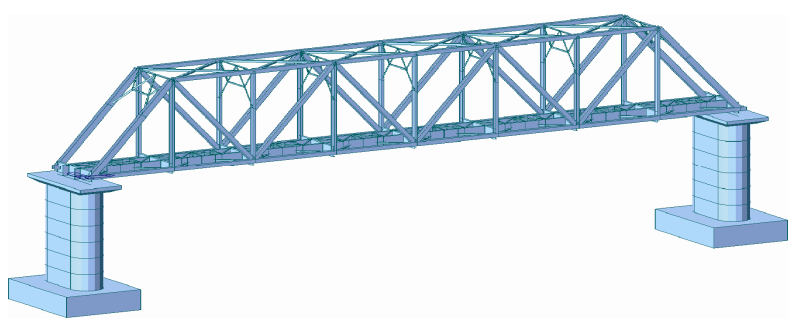

图 8 桥梁有限元模型

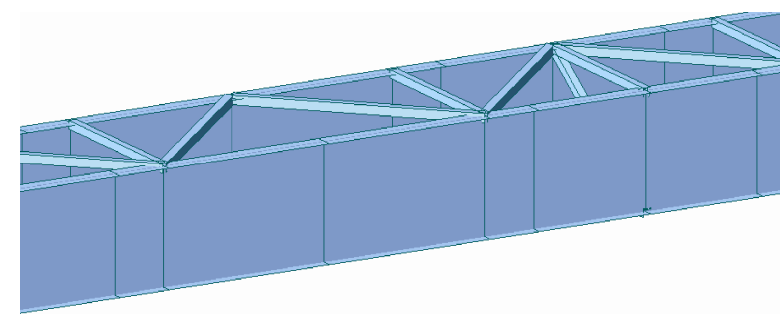

图 9 桥面有限元模型

车-线-桥系统以钢轨为界, 其上为车辆子系统, 其下为轨桥子系统。车辆系统和轨桥系统之间的耦 合关系, 体现在轮轨之间的轮轨力。本文采用赫兹 非线性弹性接触理论来描述轮轨接触弹簧。根据赫 兹理论, 轮轨垂向作用力 $N_{z}(t)$ 与轮轨间弹性压缩量 间有如下关系

$$
N_{z}(t)=\left[\frac{1}{G} \delta Z(t)\right]^{3 / 2}
$$

式中 $G$ 一一轮轨接触常数 $\left(\mathrm{m} / \mathrm{N}^{2 / 3}\right)$;

$\delta Z(t)$ 一一轮轨间的弹性压缩量 $(\mathrm{m})$ 。

建立车线桥系统运动微分方程后, 采用 Newmark- $\beta$ 法进行数值迭代求解。

\section{2 车-线-桥垂向耦合动力学分析}

\section{1 桥梁自振频率}

由 MIDAS 软件计算出的钢桥的前 3 阶自振频 率计算结果见表 4，振型见图 10 12。

表 4 钢桥的前五阶自振频率

$\mathrm{Hz}$

\begin{tabular}{cccc}
\hline & 一阶 & 二阶 & 三阶 \\
\hline 自振频率 & 1.809 & 3.179 & 3.928 \\
振型 & 对称横弯 & 横弯+扭转 & 对称坚弯 \\
\hline
\end{tabular}

桥梁的前三阶振型如下。 


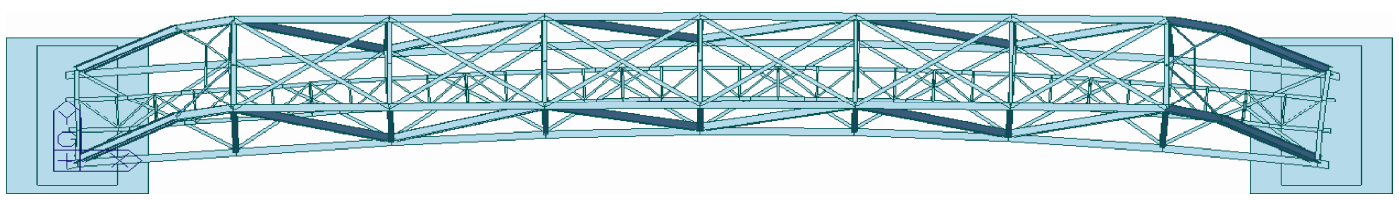

图 10 第 1 阶振型

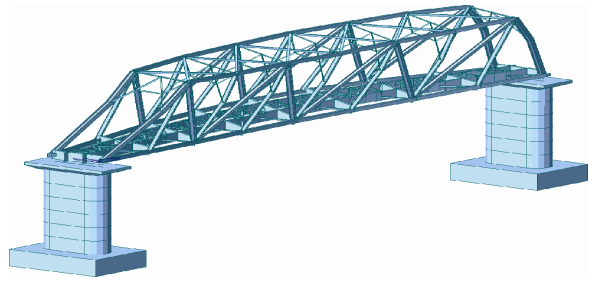

图 11 第 2 阶振型

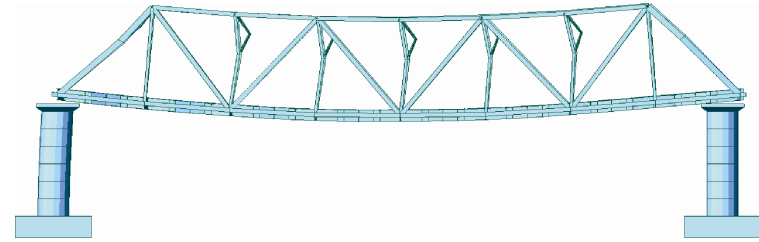

图 12 第 3 阶振型

\section{2 系统动力响应分析}

为了全面地分析复合轨枕是否满足安全要求, 对采用木枕及复合材料轨枕情况下的车线桥动力响 应进行了计算, 计算结果见表 5 8, 当列车以 80 $\mathrm{km} / \mathrm{h}$ 通过桥梁, 且铺装复合材料轨枕时, 车辆、线 路和桥梁的响应时程曲线如图 13 18 所示。依据我 国《铁道车辆动力学性能评定和试验鉴定规范》 $\left(G B\right.$ 5599-1985) ${ }^{[15]}$, 安全性指标中的轮重减载率容 许限度不大于 0.6 ; 运行平稳性指标中货车车体垂向 振动加速度的限值为 $a \leqslant 0.7 g$; 参照《铁路桥涵检定 规范》 ${ }^{[16]}$ 及 UIC 规范, 对于无碴轨道, 桥面垂向加 速度 $a_{\max } \leqslant 0.5 g$ 。

表 5 不同轨枕条件下桥梁动力响应

\begin{tabular}{|c|c|c|c|c|c|c|c|c|c|c|c|c|c|}
\hline \multirow{3}{*}{ 轨枕 } & \multirow{3}{*}{$\begin{array}{l}\text { 车速/ } \\
(\mathrm{km} / \mathrm{h})\end{array}$} & \multicolumn{6}{|c|}{ 位移/mm } & \multicolumn{6}{|c|}{ 加速度 $/\left(\mathrm{m} / \mathrm{s}^{2}\right)$} \\
\hline & & \multicolumn{3}{|c|}{ 下弦 } & \multicolumn{3}{|c|}{ 铁路纵梁 } & \multicolumn{3}{|c|}{ 下弦 } & \multicolumn{3}{|c|}{ 铁路纵梁 } \\
\hline & & 梁端 & 端横梁 & 跨中 & 梁端 & 端横梁 & 跨中 & 梁端 & 端横梁 & 跨中 & 梁端 & 端横梁 & 跨中 \\
\hline \multirow{4}{*}{ 木枕 } & 60 & 1.129 & 0.084 & 47.901 & 1.278 & 1.550 & 49.580 & 0.180 & 0.005 & 0.727 & 0.430 & 0.264 & 0.869 \\
\hline & 70 & 1.300 & 0.090 & 59.187 & 1.458 & 2.122 & 61.302 & 0.322 & 0.013 & 2.283 & 0.621 & 0.614 & 2.445 \\
\hline & 80 & 1.204 & 0.091 & 53.588 & 1.336 & 1.616 & 55.463 & 0.408 & 0.011 & 2.347 & 0.541 & 0.661 & 2.684 \\
\hline & 90 & 1.239 & 0.102 & 55.352 & 1.244 & 2.029 & 57.244 & 0.453 & 0.019 & 2.280 & 0.824 & 0.822 & 3.038 \\
\hline \multirow{4}{*}{$\begin{array}{l}\text { 复合材 } \\
\text { 料轨枕 }\end{array}$} & 60 & 1.128 & 0.084 & 47.921 & 1.278 & 1.552 & 49.570 & 0.173 & 0.005 & 0.739 & 0.439 & 0.261 & 0.876 \\
\hline & 70 & 1.301 & 0.090 & 59.192 & 1.460 & 2.130 & 61.311 & 0.344 & 0.013 & 2.326 & 0.638 & 0.671 & 2.482 \\
\hline & 80 & 1.207 & 0.091 & 53.663 & 1.340 & 1.617 & 55.540 & 0.419 & 0.012 & 2.374 & 0.640 & 0.685 & 2.707 \\
\hline & 90 & 1.236 & 0.102 & 55.387 & 1.242 & 2.030 & 57.275 & 0.484 & 0.018 & 2.223 & 0.673 & 0.812 & 2.972 \\
\hline
\end{tabular}

表 6 不同轨枕条件下钢轨振动响应

\begin{tabular}{|c|c|c|c|c|c|c|c|}
\hline \multirow{2}{*}{ 轨枕 } & \multirow{2}{*}{$\begin{array}{l}\text { 车速/ } \\
(\mathrm{km} / \mathrm{h})\end{array}$} & \multicolumn{3}{|c|}{ 位移/mm } & \multicolumn{3}{|c|}{ 加速度 $/\left(\mathrm{m} / \mathrm{s}^{2}\right)$} \\
\hline & & 过渡段 & 梁端区域 & 桥上 & 过渡段 & 梁端区域 & 桥上 \\
\hline \multirow{4}{*}{ 木枕 } & 60 & 1.720 & 3.711 & 49.978 & 0.700 & 0.828 & 1.822 \\
\hline & 70 & 2.168 & 3.501 & 62.084 & 1.502 & 1.439 & 3.655 \\
\hline & 80 & 1.958 & 3.447 & 56.398 & 1.523 & 1.141 & 2.867 \\
\hline & 90 & 2.036 & 3.953 & 57.493 & 1.979 & 1.817 & 3.836 \\
\hline \multirow{4}{*}{ 复合材料轨枕 } & 60 & 1.721 & 3.713 & 49.972 & 0.699 & 0.827 & 1.830 \\
\hline & 70 & 2.167 & 3.508 & 62.112 & 1.505 & 1.471 & 3.649 \\
\hline & 80 & 1.962 & 3.458 & 56.428 & 1.525 & 1.175 & 2.903 \\
\hline & 90 & 2.042 & 3.955 & 57.526 & 1.989 & 1.830 & 3.808 \\
\hline
\end{tabular}


表 7 不同轨枕条件下轨枕振动响应

\begin{tabular}{|c|c|c|c|c|c|c|c|}
\hline \multirow{2}{*}{ 轨枕 } & \multirow{2}{*}{$\begin{array}{l}\text { 车速/ } \\
(\mathrm{km} / \mathrm{h})\end{array}$} & \multicolumn{3}{|c|}{ 位移 $/ \mathrm{mm}$} & \multicolumn{3}{|c|}{ 加速度 $/\left(\mathrm{m} / \mathrm{s}^{2}\right)$} \\
\hline & & 过渡段 & 梁端区域 & 桥上 & 过渡段 & 梁端区域 & 桥上 \\
\hline \multirow{4}{*}{ 木枕 } & 60 & 1.133 & 2.541 & 49.888 & 0.490 & 0.513 & 1.657 \\
\hline & 70 & 1.479 & 3.114 & 61.574 & 1.051 & 0.955 & 4.062 \\
\hline & 80 & 1.302 & 2.668 & 55.856 & 1.045 & 0.959 & 2.644 \\
\hline & 90 & 1.357 & 3.187 & 57.347 & 1.341 & 1.450 & 3.347 \\
\hline \multirow{4}{*}{ 复合材料轨枕 } & 60 & 1.135 & 2.545 & 49.911 & 0.489 & 0.515 & 1.642 \\
\hline & 70 & 1.481 & 3.132 & 61.589 & 1.052 & 0.977 & 4.103 \\
\hline & 80 & 1.306 & 2.672 & 55.878 & 1.046 & 1.029 & 2.680 \\
\hline & 90 & 1.358 & 3.190 & 57.380 & 1.344 & 1.474 & 3.336 \\
\hline
\end{tabular}

表 8 不同轨枕条件下车辆振动响应

\begin{tabular}{cccc}
\hline 轨枕 & 车速 $/(\mathrm{km} / \mathrm{h})$ & 车体加速度 $/\left(\mathrm{m} / \mathrm{s}^{2}\right)$ & 轮重减载率 \\
\hline \multirow{4}{*}{ 木枕 } & 60 & 2.947 & 0.408 \\
& 70 & 5.087 & 0.495 \\
& 80 & 4.482 & 0.503 \\
\hline \multirow{2}{*}{ 复合材料 } & 90 & 5.584 & 0.571 \\
轨枕 & 60 & 3.047 & 0.410 \\
& 80 & 5.136 & 0.511 \\
& 90 & 4.516 & 0.502 \\
\hline
\end{tabular}

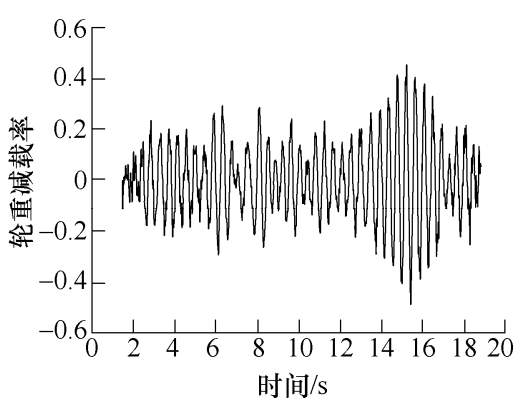

图 13 货车轮重减载率时程曲线

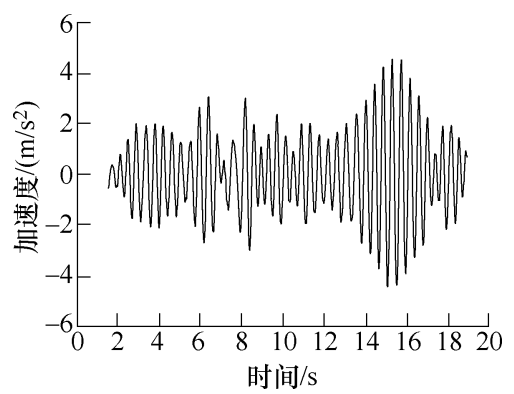

图 14 货车车体加速度时程曲线

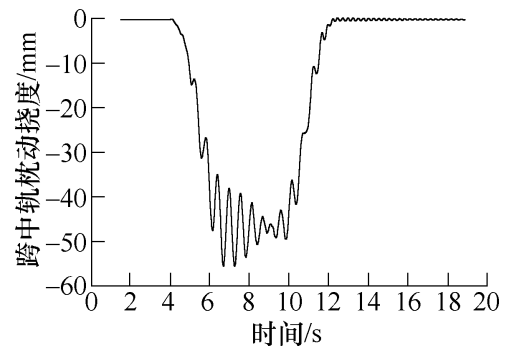

图 15 桥梁跨中轨枕动挠度时程曲线

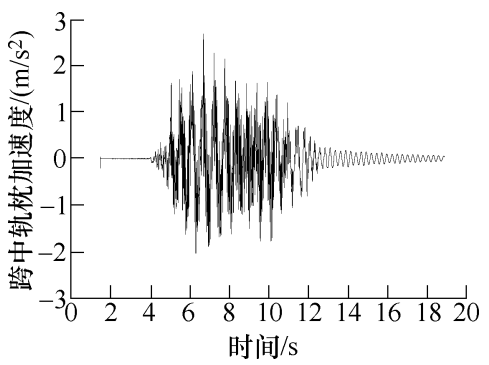

图 16 桥梁跨中轨枕加速度时程曲线

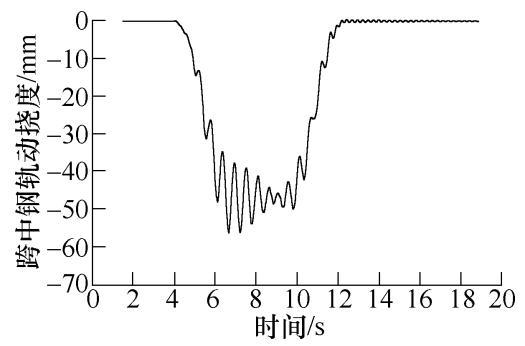

图 17 桥梁跨中钢轨位移时程曲线

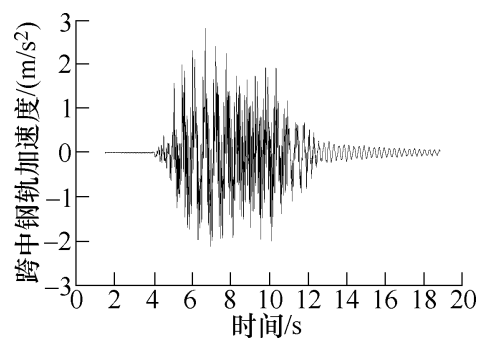

图 18 桥梁跨中钢轨加速度时程曲线

由前述计算结果可知, 铺装木枕和复合材料轨 枕时, 桥梁和车辆的动力响应均满足限值要求, 桥 梁和线路的最大响应出现于货车 $70 \mathrm{~km} / \mathrm{h}$ 通过桥梁 时, 桥梁跨中最大动挠度达到 $61.311 \mathrm{~mm}$ 。采用复 合材料轨枕替换木枕后, 钢轨、轨枕、桥梁和车 辆的动力响应数值相近, 以 $70 \mathrm{~km} / \mathrm{h}$ 过桥时的系统 动力响应为例, 桥梁位移变化最大处均为铁路纵 梁的端横梁, 增大 $3.6 \%$, 其他位置处的变化均小 于 $1 \%$; 桥梁加速度变化最大处也是铁路纵梁的端 横梁, 增大 $9.3 \%$; 不同位置处的钢轨位移变化均 小于 $1 \%$; 钢轨加速度变化最大处为梁端区域, 增 大 $2.2 \%$; 轨枕位移变化均小于 $1 \%$; 轨枕加速度变 
化最大处为梁端区域, 增大 $2.3 \%$; 车辆加速度增 大 $9.6 \%$; 轮重减载率增大 $3.2 \%$; 两种轨枕条件下 的系统动力响应接近, 主要由于复合材料轨枕设 计时即采取了刚度等效原则, 避免了刚度突变对 行车性能的影响。

\section{3 复合材料轨枕和木枕力学性能对比 分析}

采用 ABAQUS 软件建立轨枕-纵梁有限元模 型, 见图 19, 对复合材料轨枕和木枕进行局部应力 的对比分析, 以考察其是否满足要求。复合材料轨 枕和木枕均采用实体单元建模; 根据不同工况的连 接方式, 设置不同的边界条件, 固结时采用 Tie 连 接处理, 弹性连接时采用 Connector 连接处理; 纵 梁底部固结处理; 外部荷载为单根轴质量 $30 \mathrm{t}$, 作 用在钢轨上。

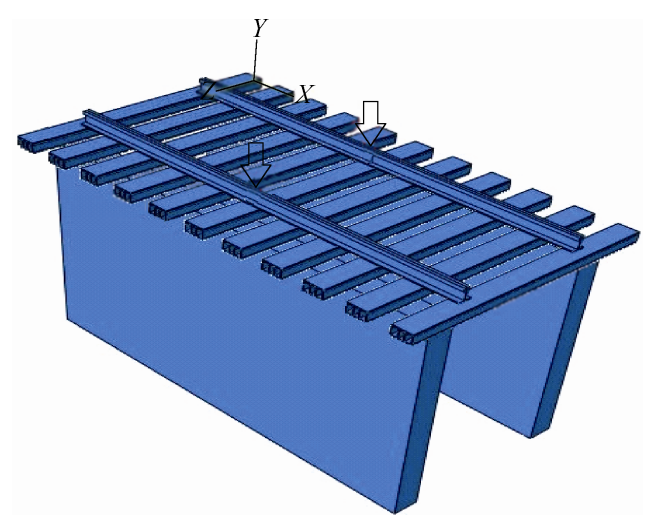

图 19 轨枕-纵梁有限元模型

\section{1 力学响应}

分别考虑连接关系、钢轨和轨枕连接刚度、轨 枕和纵梁连接刚度等因素对系统的影响, 建立 6 种
工况: (1) 钢轨与木枕固结, 木枕固支于铁路纵梁; (2) 钢轨与复合材料轨枕固结, 复合材料轨枕固支于 铁路纵梁; (3) 钢轨与木枕弹性联结, 木枕固支于铁 路纵梁; (4) 钢轨与复合材料轨枕弹性联结, 复合材 料轨枕固支于铁路纵梁; (5) 钢轨与木枕弹性联结, 木枕简支于铁路纵梁; (6) 钢轨与复合材料轨枕弹性 联结, 复合材料轨枕简支于铁路纵梁; 木枕和复合 材料轨枕应力和变形对比, 见表 9 。

表 9 木枕和复合材料轨枕对比

\begin{tabular}{|c|c|c|c|c|c|c|}
\hline & (1) & (2) & (3) & (4) & (5) & (6) \\
\hline 计算 & & 复合 & & 复合 & & 复合 \\
\hline \multirow[t]{2}{*}{ 工况 } & 木枕 & 材料 & 木枕 & 材料 & 木枕 & 材料 \\
\hline & & 轨枕 & & 轨枕 & & 轨枕 \\
\hline $\begin{array}{l}\text { 应力/ } \\
\mathrm{MPa}\end{array}$ & 2.69 & 23.42 & 1.17 & 30.98 & 1.51 & 29.65 \\
\hline $\begin{array}{c}\text { 变形/ } \\
\mathrm{mm}\end{array}$ & 0.66 & 0.49 & 0.85 & 0.64 & 1.63 & 0.97 \\
\hline
\end{tabular}

由表 9 可知，不同工况下木枕的应力均小于许 应应力 $12 \mathrm{MPa}^{[17]}$, 而复合材料轨枕的应力比木枕的 应力要大, 但是远小于复合材料轨枕的许用应力 $300 \mathrm{MPa}^{[18]}$, 主要是由于复合材料轨枕的惯性矩比 木枕的小。在实际应用过程中, 木枕可以满足重载 运输需求, 而复合材料轨枕设计时, 截面中间的两 根坚条作为加强筋, 使截面形状更加合理, 进而实 现复合材料轨枕与木枕的刚度相近替换, 计算结果 也表明两种轨枕变形相当。

\section{2 轮载分配}

列车轮载作用于钢轨时, 桥枕不是单根受力, 而是相邻多根桥枕共同承载, 只是不同桥枕分担轮 载的比例不同而已。为了研究木枕和复合材料轨枕 在连续有效和隔根失效情况下桥枕对轮载分配的差 别, 各种轨枕的轮载分配情况见表 10 。

表 10 两种工况下的轮载分配

\begin{tabular}{|c|c|c|c|c|c|c|c|c|c|}
\hline \multirow{3}{*}{$\begin{array}{l}\text { 桥枕 } \\
\text { 位置 }\end{array}$} & \multicolumn{4}{|c|}{ 轨枕连续有效 } & \multicolumn{5}{|c|}{ 轨枕隔根失效 } \\
\hline & \multicolumn{2}{|c|}{ 木枕 } & \multicolumn{2}{|c|}{ 复合材料轨枕 } & & \multicolumn{2}{|c|}{ 木枕 } & \multicolumn{2}{|c|}{ 复合材料轨枕 } \\
\hline & 分配系数 & 荷载值/kN & 分配系数 & 荷载值/kN & & 分配系数 & 荷载值/kN & 分配系数 & 荷载值 $/ \mathrm{kN}$ \\
\hline $\mathrm{R}_{1}$ & 0.213 & 31.44 & 0.226 & 33.65 & $\mathrm{R}_{1}$ & 0.369 & 53.43 & 0.378 & 54.99 \\
\hline $\mathrm{R}_{2}$ & 0.184 & 27.05 & 0.187 & 27.80 & $\mathrm{R}_{2}$ & 0.241 & 34.87 & 0.243 & 35.30 \\
\hline $\mathrm{R}_{3}$ & 0.128 & 18.78 & 0.129 & 19.19 & $\mathrm{R}_{3}$ & 0.074 & 10.75 & 0.068 & 9.93 \\
\hline $\mathrm{R}_{4}$ & 0.082 & 12.09 & 0.071 & 10.64 & - & - & - & - & - \\
\hline 合计 & 0.982 & 147.28 & 0.993 & 148.91 & 合计 & 0.964 & 144.67 & 0.970 & 145.45 \\
\hline
\end{tabular}

注: $R_{1}$ 位置为车轮作用轨枕位置, $R_{2} \sim R_{4}$ 依次向外延伸, 远离轮载位置。

轮载分配计算结果表明: 轮载在其作用点两侧 桥枕上对称分配; 桥枕不失效时轮载大致分配于其 作用点及两侧共 7 根桥枕, 复合材料轨枕的轮载分 配相比木枕相对集中, 其作用点及其两侧共 5 根桥
枕分配 $85.8 \%$ 的载荷，略大于木枕分配的 $83.7 \%$ 的 荷载; 桥枕隔根失效时轮载大致分配于其作用点及 两侧共 5 根桥枕(不含失效轨枕), 复合材料轨枕的 轮载配置相比木枕同样相对集中，其作用点及其两 
侧共 3 根桥枕分配 $86.4 \%$ 的载荷, 略大于木枕分配 的 $85.1 \%$ 的荷载; 轮载在桥枕上的分配系数与桥枕 截面抗弯刚度直接相关, 抗弯刚度越大, 距轮载作 用点越近的桥枕分担的轮载越大, 桥枕受力越不利。 两种工况数据对比说明, 桥枕出现吊空等失效病害 后, 单根桥枕承受的轮载会大大增加, 使桥枕受力 更不利, 相当于木枕而言, 虽然复合材料轨枕的距 作用点较近的轨枕受载较大, 但是两者之间的差距 小于 $2 \%$, 可认为两者较为一致。

\section{4 结论}

(1) 综合桥梁和车辆的动力响应, 对所分析的 $64 \mathrm{~m}$ 单线铁路钢析梁桥而言, 能够满足 $30 \mathrm{t}$ 轴质量 货车 $60 \sim 90 \mathrm{~km} / \mathrm{h}$ 范围内安全舒适运行。复合材料 轨枕和木枕相比, 刚度略大, 复合材料轨枕替换木 枕后, 系统动力响应无显著差异。

(2) 复合材料轨枕的应力比木枕大, 主要由于 复合材料轨枕的惯性矩比木枕小, 但应力数值远小 于复合材料的许用应力; 复合材料轨枕和木枕变形 相当, 主要由于复合材料轨枕截面中间的两根坚条 作为加强筋, 使截面形状更合理, 进而实现复合材 料轨枕与木枕的刚度相近。

(3) 轮载在其作用点两侧桥枕上对称分配; 轮 载分配系数与桥枕截面抗弯刚度相关, 抗弯刚度越 大, 距轮载作用点越近的桥枕分担的轮载越大, 受 力越不利。就复合材料轨枕而言, 虽然距作用点处 较近的受载比木枕大, 但是两者之差小于 $2 \%$, 可 认为两者较为一致。

(4) 对铺设木枕的既有钢桥而言, 复合材料轨 枕可通过截面设计使其在外形和重量上与木枕相 近，因此，复合材料轨枕适合作为木枕的替代品， 以解决木材用量大和养护维修繁琐的不足。

\section{参 考 文 献}

[1] MANALO A, ARAVITHAN T, KARUNASENA W, et al. A review of alternative materials for raplacingexisting timber sleepers[J]. Composite Structures, 2010, 92 : 603-611.

[2] QIAO E, DAVALOS J F, ZIPFEL M G. Modelling and optimal design of composite reinforced wood railroad crosstie[J]. Composite Structures, 1998(41): 87-96.

[3] THIERFELDER T, SANDSTROM E. The creosote content of used railway crossties as compared with European stipulations for hazardous waste[J]. Science of the Total Environ, 2008, 402(1): 106-112.
[4] GONG M, DELAHUNTY S, CHUI Y H, et al. Use of low grade hardwoods for fabricating laminated railway ties[J]. Constrution and Building Materials, 2013, 41(41): 73-78.

[5] ERP G V, MCKAY M. Recent Australian developments in fibre composite railway sleepers[J]. Electronic Journal of Structural Engineering, 2013， 13(1): 62-66.

[6] 長藤敬晴, 阿部則次. 合成まくらぎ 15 年の経験 $[\mathrm{J}]$. 鉄 道総研報告，1997，11(2)：43-48.

Keiharu Nagato, Noriji Abe. 15 Years laying experience of synthetic sleeper[J]. Railway Institute Research Report, 1997, 11(2): 43-48.

[7] TAKAI H, SATOY, SATO K. Japanese twenty five years experiences and standardization of synthetic sleeper[C]/Proceedings of 7 World Congress on Railway Research, Montreal, Canada, 2006.

[8] KOLLER G. FFU Synthetic sleepers offer material gains[J]. Railway Gazette International, 2010, 166(8) : $42-43$.

[9] OTTER D, PATTON R D, JOY R B. Developments in Alterna-tive bridge ties for open deck steel bridges[C]//2012 Annual Conference \& Exposition , Chicago, IL., American, 2012.

[10] LOPRESTI J. Fiberglass wrapped tie performance evaluation report[R]. Transportation Technology Center. Inc, 2005.

[11] LOTFY I, FARHAT M, ISSA M A, et al. Flexural behavior of high-density polyethylene railroad crossties[J]. Proceedings of the Institution of Mechanical Engineers, Journal of Rail and Rapid Transit, 2016, 230(3): 813-824.

[12] 王守琛. 新型高聚物复合材料轨枕成型技术的研究 [D]. 北京: 北京化工大学，2012.

WANG Shouchen. Study on forming technology of new polymer composite sleeper [D]. Beijing : Beijing University of Chemical Technology, 2012.

[13] 陈玉霄. 新型复合材料轨枕组分与结构设计的研究 [D]. 哈尔滨：东北林业大学，2008。

CHEN Yuxiao. Study on composition and structure design of new type of sleeper composite[D]. Haerbin: Northeast Forestry University, 2008.

[14] 杜宪亭, 夏禾, 张田. 基于精细 Runge-Kutta 混合积分 法的车桥耦合振动非迭代求解算法 [J]. 振动与冲击, 2013(13): 39-42.

DU Xianting, XIA He, ZHANG Tian. Non-iterative solving algorithm for coupled vibration of a train-bridge system based on precise Runge-Kutta hybrid integration method[J]. Journal of Vibration and Shock, 2013(13): $39-42$. 
[15] GB5599-1985, 铁道车辆动力学性能评定和试验鉴定规 范[S]. 北京: 中国标准局, 1985.

GB5599-85, Railway vehicles-specification for evaluation the dynamic performance and accreditation test[S]. Beijing: China Bureau of Standards, 1985.

[16] 铁运函[2004]120 号, 铁路桥梁检定规范[S]. 北京: 中 华人民共和国铁道部, 2004.

Railway Transport[2004]120, Code for rating existing railway bridges[S]. Beijing: Ministry of Railways of China, 2004.

[17] JTJ 025-86, 公路桥涵钢结构及木结构设计规范[S]. 北 京：人民交通出版社，1988.
JTJ 025-86, Specifications for design of steel structure and timber structure highway bridge and culvets[S]. Beijing: China Communications Press, 1988.

[18] 中国铁道科学研究院. 复合材料轨枕受力状态分析报 告[R]. 北京: 中国铁道科学研究院, 2016.

China Academy of Railway Sciences Corporation Limited. Analysis report on stress state of composite sleeper[R]. Beijing: China Academy of Railway Sciences Corporation Limited, 2016.

作者简介: 张寒, 男, 1985 年出生, 博士, 讲师。主要研究方向为车线 桥耦合振动。

E-mail: figozq100@sina.com

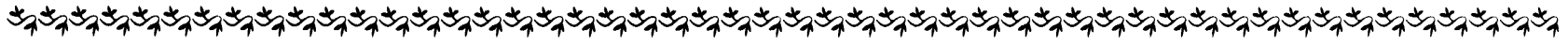

\section{(上接第 144 页)}

GONG Jianwei, JIANG Yan, XU Wei. Model predictive control for self-driving vehicles[M]. Beijing: Beijing Institute of Technology Press, 2014.

[21] 李升波, 王建强, 李克强. 软约束线性模型预测控制 系统稳定性方法[J]. 清华大学学报, 2010(11): 11481152.

LI Shengbo, WANG Jianqiang, LI Keqiang. Stability method for predictive control system of soft constrained linear model[J]. Journal of Tsinghua University, 2010(11): 1148-1152.
[22] 罗禹贡, 陈涛, 李克强. 混合动力汽车非线性模型预测 巡航控制[J]. 机械工程学报, 2015，51(16): 11-18.

LUO Yugong, CHEN Tao, LI Keqiang. Nonlinear model predictive cruise control of hybrid electric vehicle[J]. Journal of Mechanical Engineering, 2015, 51(16): 11-18.

作者简介: 王艺, 女, 1992 年出生。主要研究方向为智能车辆转向控制。 E-mail: shivery wang@163.com

蔡英风(通信作者), 女, 1985 年出生, 博士, 副教授, 硕士研究生导师。 主要研究方向为交通感知与智能车辆。

E-mail: caicaixiao0304@126.com 